\title{
A comparative study of clinical and laboratory profiles of scrub typhus in different age groups: our experience
}

\author{
Rachita Sarangi ${ }^{1}$, Shobhitendu Kabi ${ }^{2}$, Srikant Kumar Dhar' ${ }^{2}$, Avantika Dhanawat ${ }^{1}$, \\ Goutam Benia', Mahesh Chandra Sahu ${ }^{3}$ \\ 'Department of Paediatrics, IMS and SUM Hospital, Siksha 'O' Anusandhan University, K-8, Kalinga Nagar, Bhubaneswar, \\ Odisha, India \\ 2Department of Medicine, IMS and SUM Hospital, Siksha 'O' Anusandhan University, K-8, Kalinga Nagar, Bhubaneswar, \\ Odisha, India \\ 3Directorate of Medical Research, IMS and SUM Hospital, Siksha 'O' Anusandhan University, K-8, Kalinga Nagar, Bhubaneswar, \\ Odisha, India
}

\section{ABSTRACT}

Aim of the study: Rickettsial infections rate as the second most frequently reported infections for non-malarial febrile illnesses in South East Asia, after dengue. Scrub typhus is an acute infectious disease caused by Orientia tsutsugamushi, which is categorised in the Rickettsia genus.

Material and methods: This prospective study was carried out from July 2015 to June 2016 in the Department of Paediatric and Internal Medicine after obtaining institutional ethical clearance. A thorough history, meticulous physical examination, course of the hospital stay, complications, and outcomes were properly documented. Basic laboratory studies with additional investigations were performed. Serum IgM ELISA for scrub typhus was performed in all suspected cases and an optical density (OD) greater than 0.5 was considered to be positive.

Results: Scrub typhus was diagnosed in 106 cases (IgM ELISA positive) during the study period; 48 (45\%) were in the paediatric age group ( $\leq 14$ years) and 58 were adults. Gastrointestinal symptoms, hepatomegaly, and lymphadenopathy were more frequent in the paediatric age group than in adults $(p \leq 0.05)$. Eschar was found in $28.3 \%$ of cases, but there was no significant difference between paediatric and adult populations. Encephalopathy was more common in children, but other organ dysfunctions like myocarditis, renal failure, and sepsis were more common in adults $(p \leq 0.05)$.

Conclusions: Scrub typhus is seen equally in children and in adults. Clinically GI symptoms and encephalopathy are more common in children, whereas other organ dysfunctions are more common in adults. Larger multicentric studies are required to confirm our findings.

\section{KEY WORDS:}

rickettsial infection, scrub typhus, India, clinical manifestations, eschar.

\section{INTRODUCTION}

Scrub typhus is an acute infectious disease caused by Orientia tsutsugamushi, which is categorised in the Rick- ettsia genus. The organism is transmitted through the bite of larval forms (chiggers) of trombiculid mites [1]. Rickettsial infection has been reported in various parts of the world [2-8]. An estimated one billion people are at risk

\section{ADDRESS FOR CORRESPONDENCE:}

Dr. Rachita Sarangi, Professor, Department of Paediatrics, IMS and SUM Hospital, Siksha O Anusandhan

University, Bhubaneswar, Odisha, India, e-mail: rachitapaedia@gmail.com 
for scrub typhus, and around one million cases occur annually $[9,10]$. In the Indian subcontinent scrub typhus is the most commonly reported rickettsial infection [4-7, 11-13]. Scrub typhus is prevalent throughout India, particularly Southern India, Northern India, North eastern states, and central India $[12,14]$. Scrub typhus caused major epidemics resulting in significant morbidity and mortality in India during World War II [15] and thus became known as a disease associated with war. Nevertheless, there were scarcity of reports of this disease for several decades. But in view of the latest reports from various parts of India and neighbouring countries it is evident that the rickettsial infection in India has increased many-fold [16].

Rickettsial infections are caused by a distinctive group of obligate intra-cellular, Gram-negative, highly pleomorphic bacteria. This is mostly of zoonotic origin and usually transmitted to humans by arthropods like fleas, lice, mites, and ticks. The clinical manifestation of this disease varies in severity from a mild febrile illness to severe, potentially fatal disease with multiple organ dysfunction syndrome. Diagnosis of the disease should initially be based on epidemiological criteria, which include the location, travel history, season, etc. Although this infection can be easily treated, clinical diagnosis is often difficult because of its varied presentation and absence of eschar in the majority of patients. The clinical manifestations of cases reported from India show differences compared to the clinical manifestations reported from other endemic countries in Asia [17]. This could be due to difference in infecting strains, which are known to have a high level of antigenic variations. Conventional diagnostic tests such as the Weil-Felix test are insensitive and non-specific. The recently introduce serological test IgM antibody against scrub typhus (done by is ELISA or immunofluorescence assay) is considered as the gold standard $[18,19]$.

Several studies are available to describe the clinical profile, laboratory parameters, clinical predictors, and prognostic indicators both in paediatric and in adult populations. However, there is no comparative study available to describe the differences between paediatric and adult age groups. Therefore, we conducted this study in the Department of Paediatric and Internal Medicine to find out the differences in presentation and complications of this disease in children and in adults.

\section{MATERIAL AND METHODS}

This prospective study was carried out from July 2015 to June 2016 in the Department of Paediatrics and Internal Medicine after obtaining due institutional ethical clearance. Consecutive patients who were admitted with fever of more than five days duration and had clinical suspicion of scrub typhus, were enrolled as suspected cases in this study. The case definition includes patients with high-grade intermittent fever and having five out of the following eight clinical features, such as: headache, myalgia, regional lymphadenopathy, generalised lymphadenopathy, hepatomegaly, splenomegaly, eschar, and rash. A thorough history, meticulous physical examination findings, hospital course, complications (if any), and outcome were recorded in a proforma. Basic laboratory studies such as complete blood count, urine routine and microscopic examination, liver and renal function tests, and chest X-ray were performed in all cases. Additional investigations such as cerebrospinal fluid (CSF) examination, echocardiography, and radiological imaging, such as ultrasound of the abdomen and CT scan of the brain, were performed where indicated. Blood and urine culture, Quantified Buffy Coat (QBC) testing for malaria parasite, and serology for dengue and leptospirosis were done to exclude some prevalent diseases.

Serum IgM ELISA was performed in all suspected cases using scrub typhus (In Bios International Inc. [country of manufacture]) as per the manufacture's instructions. Detection of IgM antibody in ELISA for O. tsutsugamushi in serum samples with optical density (OD) more than 0.5 was considered to be a positive result for typhus and was recorded as a confirmed case of scrub typhus.

Statistical analysis was done using SPSS software version 20, the comparison of discrete variables was done by using chi-square/Fisher exact test, and continuous variables were compared using student's $t$ test. $P$-value $<0.05$ was taken as significant.

\section{OBSERVATION}

We found 106 confirmed cases of scrub typhus (IgM ELISA positive) during the study period, and out of them 48 were in the paediatric age group and 58 were adults. The mean age of children with scrub typhus was $5.3 \pm \mathrm{SD}$ years and of adults was $38.60 \pm$ SD years. There were four infants among the paediatric cases. In both groups there were more males than females, and the overall male-tofemale ratio was $2: 1$ (Table 1 ).

The major presenting symptoms were fever $(100 \%)$, gastrointestinal symptoms (70.7\%), and neurological symptoms $(60.3 \%)$. Children were more prone to abdomi-

TABLE 1. Age and gender wise distribution

\begin{tabular}{|l|c|c|c|}
\hline \multicolumn{2}{|c|}{ Children $(n=48)$} & \multicolumn{2}{c|}{ Adults $(n=58)$} \\
\hline Age (years) & $n(\%)$ & Age (years) & $n(\%)$ \\
\hline$<1$ & $4(8.3)$ & $15-30$ & $19(32.8)$ \\
\hline $1-5$ & $26(54.2)$ & $30-45$ & $23(39.7)$ \\
\hline $5-10$ & $13(27.1)$ & $45-60$ & $14(24.1)$ \\
\hline $10-15$ & $5(10.4)$ & $>60$ & $2(3.4)$ \\
\hline Gender & & & \\
\hline Male & $29(60.4)$ & Male & $42(72.4)$ \\
\hline Female & $19(39.6)$ & Female & $16(27.6)$ \\
\hline
\end{tabular}


TABLE 2. Comparison of variables

\begin{tabular}{|l|c|c|c|}
\hline Variables & $\begin{array}{c}\text { Children } \\
(n=48)\end{array}$ & $\begin{array}{c}\text { Adults } \\
(n=58)\end{array}$ & \multicolumn{1}{|c|}{$p$} \\
\cline { 2 - 3 } & $n(\%)$ & $n(\%)$ & \\
\hline $\begin{array}{l}\text { Gastrointestinal } \\
\text { symptoms }\end{array}$ & $46(95.8)$ & $29(50)$ & 0.001 \\
\hline Abdominal pain & $20(41.6)$ & $26(44.8)$ & 0.747 \\
\hline Headache and myalgia & $17(35.4)$ & $47(81)$ & 0.001 \\
\hline Rash & $5(10.4)$ & $16(27.5)$ & 0.027 \\
\hline Jaundice & $3(6.2)$ & $13(22.4)$ & 0.012 \\
\hline Lymphadenopathy & $26(54.2)$ & $15(25.8)$ & 0.003 \\
\hline Ascites & $5(10.4)$ & $3(5.1)$ & 0.314 \\
\hline Eschar & $17(35.4)$ & $13(22.4)$ & 0.142 \\
\hline Hepatomegaly & $42(87.5)$ & $25(43.1)$ & 0.001 \\
\hline Splenomegaly & $26(54.1)$ & $25(43.1)$ & 0.261 \\
\hline Edema & $17(35.4)$ & $13(22.4)$ & 0.142 \\
\hline Leucocytosis & $40(83.4)$ & $21(36.2)$ & 0.001 \\
\hline Thrombocytopenia & $6(12.5)$ & $2(3.4)$ & 0.080 \\
\hline Hypoalbuminemia & $26(54.2)$ & $29(50)$ & 0.833 \\
\hline Deranged LFT & $36(75)$ & $33(56.8)$ & 0.052 \\
\hline Deranged RFT & $4(8.3)$ & $16(27.5)$ & 0.011 \\
\hline Encephalopathy & $16(33)$ & $6(10.3)$ & 0.003 \\
\hline Pneumonia/ARDS & $13(27.1)$ & $20(34.5)$ & 0.418 \\
\hline Myocarditis & $1(2.9)$ & $30(51.7)$ & 0.001 \\
\hline Sepsis & $6(12.5)$ & $21(36.2)$ & 0.005 \\
\hline Renal failure & $4(8.3)$ & $16(27.6)$ & 0.011 \\
\hline
\end{tabular}

nal manifestations such as vomiting and pain in the abdomen, whereas adults had more neurological presentations ( $p \leq 0.05)$. Major clinical findings were hepatomegaly (63.2\%), splenomegaly (48.1\%), lymphadenopathy (38.6\%), and oedema (28.3\%). Hepatomegaly and lymphadenopathy were found to be more commonly associated findings in the paediatric age group than in adults $(p \leq 0.05)$. Eschar was found in $28.3 \%$ of cases, but there was no significant difference between paediatric and adult populations.

Leucocytosis was found to be a common laboratory finding (57.5\%), and children were more prone to it. Deranged liver function tests (65\%), pneumonia and ARDS (31.1\%), myocarditis (29.2\%), sepsis (25.4\%), and renal failure (18.8\%) were common complications found in our study. Encephalopathy was found to be more common in children, but the other organ dysfunctions such as cardiac and renal were more commonly associated with the adult age group ( $p \leq 0.05$; Table 2 ).

\section{DISCUSSION}

The overall burden of the disease is greater in the rural population of Asia, with studies showing scrub typhus being responsible for up to $20 \%$ of all hospital admissions [20]. This disease has previously been found to account for up to $50 \%$ of undifferentiated febrile illness occurring in southern India, particularly in cooler months of the year [21]. This was a prospective study carried out in a tertiary care hospital in Odisha, Eastern India, which has a large catchment area for referral patients. To our surprise, we found four infants in our study population of 106 cases of scrub typhus. It is likely that these infants were exposed to trombiculid mites while they were carried to the working field by their mothers.

The severity of illness depends on both host- and pathogen-related factors. Pathogen-related factors may be attributed to the fact that different strains of $O$. tsutsugamushi may contribute differently to the manifestation of the disease. On the other hand, the difference in clinical response of the host might be due to their immunological response, where one group is immunologically virgin to the strain of $O$. tsutsugamush $i$ and others are indigenous population partially immune due to repeated subclinical infections, or the infective strain may have been different. Hence, the manifestations vary from mild and self-limiting to fatal complications. Most of the patients presented with fever (100\%), GI symptoms (70.7\%), and neurological manifestations $(60.3 \%)$, as in other studies [7, 22-24], with mean duration of fever of 7.4 days. Headache and myalgia were found to be more prevalent among adults $(p \leq 0.05)$, perhaps because of more expressive capability and psychosomatic reaction than the paediatric population. Eschar was found in $28.3 \%$ of patients, and there was no significant variation in its occurrence among adults and in children. However, there was a significant variation in its prevalence because Mahajan et al. found it in only 9.5\% in North India [25], whereas in Korea eschar was documented in as many as $90 \%$ of the patients [26]. The variation in the prevalence of eschar may be because of variation in the strains of organism, variation in cutaneous immunity among patients, or a variable search of eschar in different studies. Hepatomegaly (63.2\%), splenomegaly (48.1\%), and lymphadenopathy (38.6\%) were found in a significantly higher percentage of patients in our study. Moreover, hepatomegaly and lymphadenopathy were found to be more common findings among children than among adults as a marker of strong systemic inflammation. Many patients were found to have multisystem involvement, like liver dysfunction, renal impairment, ARDS, meningoencephalitis, and overlapping sepsis. MODS were found more commonly in adults than in children. This could be attributed to their advancing age and the existence of other co-morbid conditions like alcoholism, diabetes mellitus, COPD, coronary artery disease, and chronic kidney disease, which can predispose them to higher incidence of organ dysfunction. Scrub meningoencephalitis was a more common finding among children than in adults, which can be an issue of concern regarding damage to the developing brain and having a greater number of sequalae. 
There are certain limitations to this study. It could not be a proper reflection of prevalence of the disease and its manifestations in the community because it was a single centre, hospital-based study. Although it has a large catchment area of referral, the exact burden of the disease may be much higher in the community. In the adult survey the pre-existing co-morbid conditions were not recorded properly, which can reflect as more multiorgan dysfunction among adults compared to children.

\section{CONCLUSIONS}

Physicians should consider scrub typhus as a close differential diagnosis when caring for patients with undifferentiated acute febrile illness with multisystem involvement. A high index of suspicion, rapid diagnosis, and early start of appropriate therapy can curtail the morbidity and mortality. Strain variations may contribute to the variations to the disease manifestations, hence further epidemiological and molecular studies are required to identify the prevalent strains and their antigenic variations in this part of the country. Larger multicentre studies may be carried out to ascertain the endemicity, variation in clinical manifestations, prognostic indicators, and predictive value of signs and symptoms in different age groups.

\section{DISCLOSURE}

The authors declare no conflict of interest.

\section{REFERENCES}

1. Mahajan SK. Scrub typhus. J Assoc Physicians India 2005; 53: 954-258.

2. Panpanich R, Garner P. Antibiotics for treating scrub typhus. Cochrane database Syst Rev 2002; 3: CD002150.

3. Huang CT, Chi H, Lee HC, et al. Scrub typhus in children in a teaching hospital in eastern Taiwan, 2000-2005. Southeast Asian J Trop Med Public Health 2009; 40: 789-794.

4. Sirisanthana V, Puthanakit T, Sirisanthana T. Epidemiologic, clinical and laboratory features of scrub typhus in thirty Thai children. Pediatr Infect Dis J 2003; 22: 341-345.

5. Chanta C, Chanta S. Clinical study of 20 children with scrub typhus at Chiang Rai Regional Hospital. J Med Assoc Thai 2005; 88: 18671872.

6. Silpapojakul K, Chupupakkarn S, Yuthasompob S, et al. Scrub and murine typhus in children with obscure fever in the tropics. Pediatr Infect Dis J 1991; 10: 200-203.

7. Mahajan SK, Rolain JM, Sankhyan N, et al. Pediatric scrub typhus in Indian Himalayas. Indian J Pediatr 2008; 75: 947-949.

8. Lee CS, Min IS, Hwang JH, et al. Clinical significance of hypoalbuminemia in outcome of patients with scrub typhus. BMC Infect Dis 2010; 10: 216-220

9. Kelly DJ, Fuerst PA, Ching WM, Richards AL. Scrub typhus: the geographic distribution of phenotypic and genotypic variants of Orientia tsutsugamushi. Clin Infecti Dis 2009; 48: 203-230.

10. Watt G, Paro P. Scrub typhus and tropical Rickettsioses. Curr Opin Infect Dis 2003; 16: 429-436.
11. Vivekanandan M, Mani A, Priya YS, et al. Outbreak of scrub typhus in Pondicherry. J Assoc Physicians India 2010; 58: 24-28.

12. Sharma A, Mahajan S, Gupta ML, et al. Investigations of an outbreak of scrub typhus in the Himalayan Region of India. Jpn Infect Dis 2005; 58: 208-210.

13. Razak A, Sathyanarayanan V, Prabhu M, et al. Scrub typhus in Southern India: are we doing enough. Trop Doct 2010; 40: 149-151.

14. Rathi NB, Rathi AN, Goodman MH, Aghai ZH. Rickettsia diseases in India:proposed clinical scoring systems for early detection of spotted fever. Ind Paediatric 2011; 48: 867-872.

15. Sayen JJ, Pond HS, Forrester JS, Wood FC. Scrub typhus in Assam and Burma: clinical study of 616 cases. Medicine (Baltimore) 1946; 25: $155-214$.

16. Vivekanandan M, Nayar I, Remalayam B, George T. Scrub typhus meningitis in South India - a retrospective study. PLoS One 2013; 8: 66595.

17. Varghese GM, Abraham OC, Mathai D, et al. Scrub typhus among hospitalized patients with febrile illness in South India: magnitude and clinical predictors. J Infect 2006; 52: 56-60.

18. Wong SY, Lam MS. Rickettsioses: the new and old diseases. Singapore Med J 2001; 42: 546.

19. Chenchittikul M, Khlumklai S, Saisongkoth W, Bhumisawasdi J. Comparison of the Weil Felix (Proteus mirabilis OXK) test and indirect fluorescent antibody test for serodiagnosis of scrub typhus. J Med Tech Assoc Thai 1995; 23: 55-63.

20. Brown GW, Robinson DM, Huxsoll DL, et al. Scrub typhus: a common cause of illness in indigenous population. Trans R Soc Trop Med Hyg 1976; 70: 444-448.

21. Issac R, Varghese GM, Mathai E, et al. Scrub typhus: prevalence and diagnostic issues in rural southern India. Clin Infect Dis 2004; 39 : 1395-1396.

22. Kumar M, Krishnamurthy S, Delhikumar CG, et al. Scrub typhus in children at a tertiary hospital in southern India: clinical profile and complications. J Infect Public Health 2012; 5: 82-88.

23. Sankhyan N, Saptharishi LG, Sasidaran K, et al. Clinical profile of scrub typhus in children and its association with hemophagocytic lymphohistiocytosis. Indian Pediatr 2014; 51: 651-653.

24. Gupta N, Mittal V, Gurung B, Sherpa U. Pediatric scrub typhus in South Sikkim. Indian Pediatr 2012; 49: 322-324.

25. Mahajan SK, Rolain JM, Kashyap R, et al. Scrub typhus in Himalayas. Emerg Infect Dis 2006; 12: 1590-1592.

26. Kim DM, Kim SW, Choi SH, Yun NR. Clinical and laboratory findings associated with severe scrub typhus. BMC Infect Dis 2010; 10: 108. 